\title{
Non-Cytotoxic Agent
}

National Cancer Institute

\section{Source}

National Cancer Institute. Non-Cytotoxic Agent. NCI Thesaurus. Code C83487.

A pharmacologic substance that does not directly kill cells, but instead affects cellular transport and metabolic functions to ultimately produce cell death. 